\title{
DEVELOPMENT OF ARTIFICIAL NEURAL NETWORK MODEL IN PREDICTING PERFORMANCE OF THE SMART WIND TURBINE BLADE
}

\author{
E.E. Supeni ${ }^{1}$, J.A. Epaarachchi ${ }^{2}$, M.M. Islam ${ }^{2}$ and K.T. Lau ${ }^{2,3}$ \\ ${ }^{1}$ Department of Mechanical Engineering, Faculty of Engineering, \\ Universiti Putra Malaysia, Malaysia \\ ${ }^{2}$ Centre Excellence in Engineered Fibre Composites, \\ University of Southern Queensland, Australia \\ ${ }^{3}$ Department of Mechanical Engineering, \\ Hong Kong Polytechnic University HongKong, China \\ E-mail: ErisElianddy.Supeni@usq.edu.au
}

\begin{abstract}
This paper demonstrates the applicability of artificial neural networks (ANNs) that use multiple bck-propagation networks (MBP) and a non-linear autoregressive exogenous model (NARX) for predicting the deflection of a smart wind turbine blade specimen. A neural network model has been developed to perform the deflection with respect to the number of wires required as the output parameter, and parameters such as load, current, time taken and deflection as the input parameters. The network has been trained with experimental data obtained from experimental work. The various stages involved in the development of a genetic algorithm based neural network model are addressed in detail in this paper.
\end{abstract}

Keywords: Artificial neural network; back-propagation; multiple back-propagation; non-linear autoregressive exogenous model.

\section{INTRODUCTION}

Glass fiber reinforced polymer (GFRP) is a promising material for renewable energy which has been mainly used in wind turbine blades, due to its high and strength weight ratio (Aeyzarq Muhammad Hadzreel \& Siti Rabiatull Aisha, 2013; M. Khairul Zaimy, Zafiah, Rus, Ab Latif, \& Nurulsaidatulsyida, 2013; Nolet, 2011). The application of GFRP laminates improves the ultimate strength in capturing wind as a proportion of the diameter of the blade (Sorensen, Jørgensen, Christian, \& Jensen, 2004). However, a longer blade will result in deflection since the bending moment is high from the tip to root. At this point, there is more blade outboard (contributing to bending moment) than at any other point along the blade (Peter \& Richard, 2012). At the tip the bending moment drops to zero, as shown in Figure 1.The nature of the composite material is a high non-linear system. It is a crucial problem to describe the characteristics of composite load deflection. To alleviate the deflection of the GFRP, it will be enhanced to use shape memory alloy (SMA) wires (Supeni, Epaarachchi, Islam, \& Lau, 2012a, 2012b). As ANNs have a strong ability to describe non-linear mapping, there are many uses in load modeling studies, and researchers are trying to describe the complex characteristics of the performance of the composite load precisely (Sapuan \& Iqbal, 2010). The neural network is used in the parameter identification of traditional models such as difference equations models, power function models and polynomial function models, but the BP neural network is only used as an optimization algorithm, and the 
structure of the models has not improved, owing to disadvantages such as slow convergence and local minimum (Gayan, Jayantha, Hao, \& Lau, 2013). The performance of GFRP-plated specimens depends upon the load applied, the internal structure of the SMA and the current applied. This paper presents the results of experimental investigations carried out on 6 SMA wires used to reinforce a rectangular plate along with an artificial neural network (ANN) based model for performance prediction.

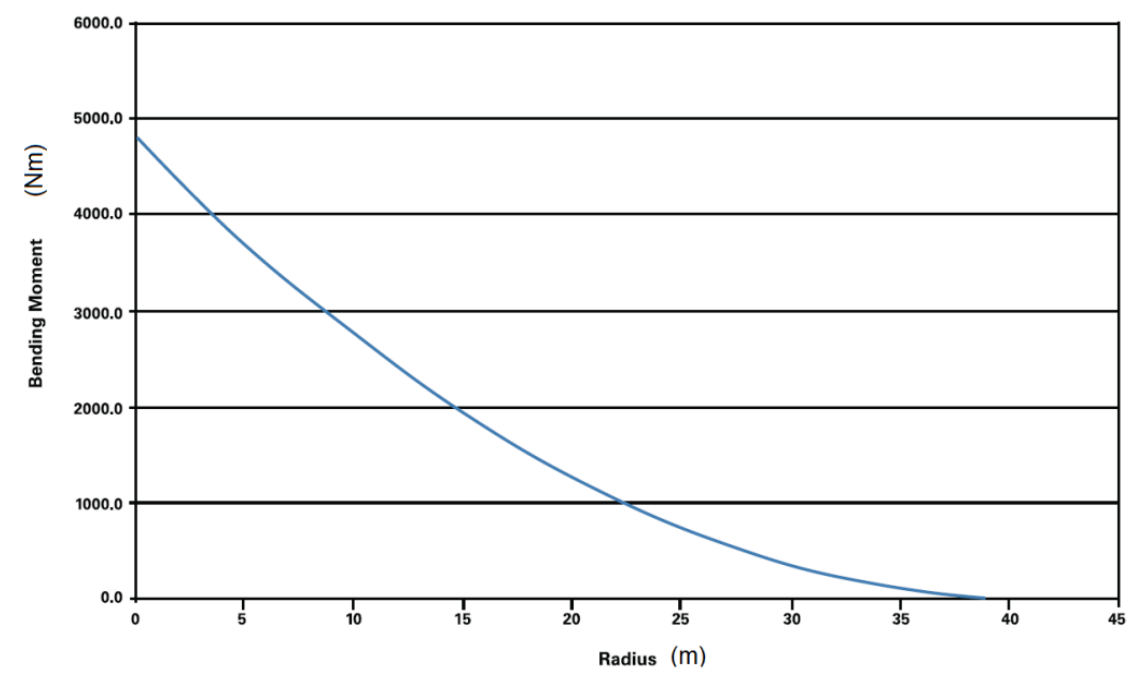

Figure 1.Bending moment against radius in a large turbine blade (Nolet, 2011)

\section{RESEARCH SIGNIFICANCE}

The effect of the current applied in the SMA wires and correlation of the deflection of the plate has been modeled in ABAQUS in Figure 2 and tested experimentally in Figure 3 (Abaqus, 2012). The results obtained from the investigation were used to generate an ANN-based design tool for predicting the amount of wire needed to restore the original shape of such bending. This depends on parameters such as deflection, the total current and the applied load.

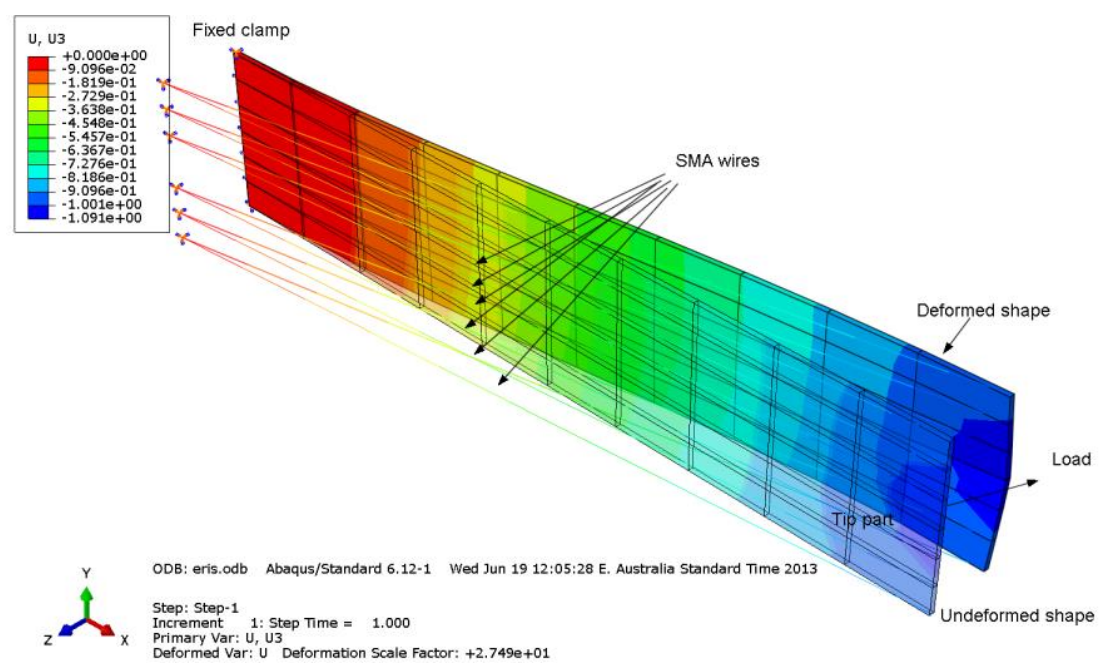

Figure 2.The plate simulated in ABAQUS FEA 


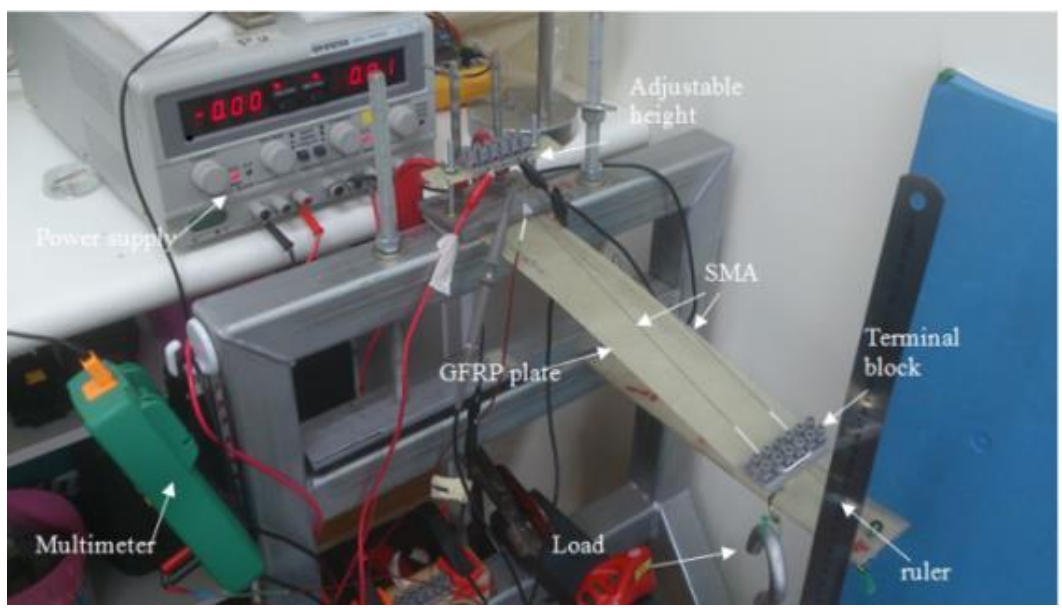

Figure 3.Photograph of tested composite plate.

\section{METHODOLOGY}

This study is to evaluate the predictive ability using machine learning (ML) based on MBP and NARX. The performance of MBP and NARX is compared. To facilitate the comparison of the performance, all the networks simulated have been designed and trained accordingly, from input layers, hidden layers and output layers. Output neurons use hyperbolic tangent activation functions. The standard back-propagation algorithm is used to train the networks with a learning rate equal to 0.01 .Once a given network has been trained, it is required to provide estimates of the future sample values of a given time series for a certain prediction. The predictions are executed in a recursive curve until the desired prediction horizon is reached, i.e., during $\mathrm{N}$ time steps the predicted values are fed back in order to take part in the composition of the regressors. The networks are evaluated in terms of the root mean square error (RMSE). Parameters such as applied load (L), applied current (I) and deflection (d) have been used as input and the number of wires (NW) as the output for the designed ANN. The general schematic diagram is illustrated in Figure 4.

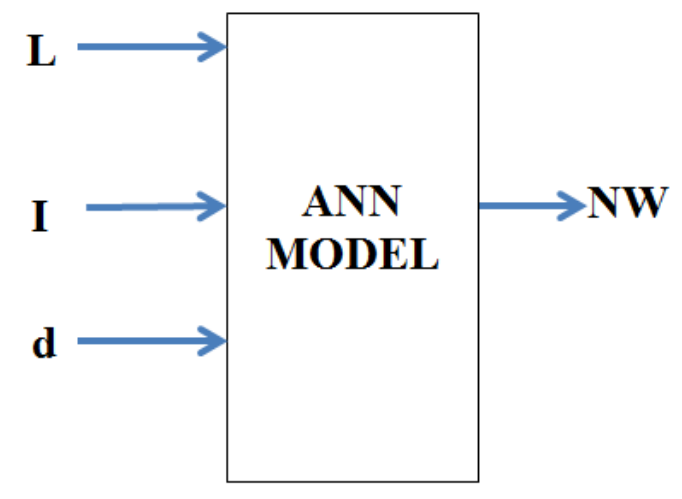

Figure 4.General structure of ANN model.

The network structure of the proposed ANN was divided into three randomly selected batches: the training batch, testing batch and validation batch. The regression analysis capacity of the network could be checked after the training phase. The mean square error (MSE), determination coefficient $\mathrm{R}$ and root mean square errors (RMSE) 
are measured by the suggested neural network. In total, 162 data sets were selected for this designed ANN and are broken down into each target time step as follows: 130, 16 and 16 for training, testing and validation respectively.

\section{MBP Method}

Figure 5 illustrates the learning process of a multi-layer neural network employing a back-propagation algorithm. To illustrate this process, a three-layer neural network with, for example, three inputs, three hidden layers and one output is implemented. Two types of sigmoid activation functions are selected for several numbers of hidden, output layer 2, which are the logarithmic sigmoid function (logsig) and the hyperbolic tangent sigmoid function (tansig) respectively. The adjustable weights that are used to propagate errors back are equal to this output value used during computing. Only the direction of the data flow is changed (signals are propagated from output to inputs one after the other). This technique is used for all network layers. For comparative study, a free open source software was used to generate the MBP which uses program code C (Khan, Rahman, Kadirgama, \& Bakar, 2012; Khan, Rahman, Kadirgama, Maleque, \& Bakar, 2011; Noel \& Bernardete, 2001; Noel \& Bernardete, 2003; Rahman, Mohyaldeen, Noor, Kadirgama, \& Bakar, 2011; Srihari, Govindarajulu, \& Ramachandra, 2010).

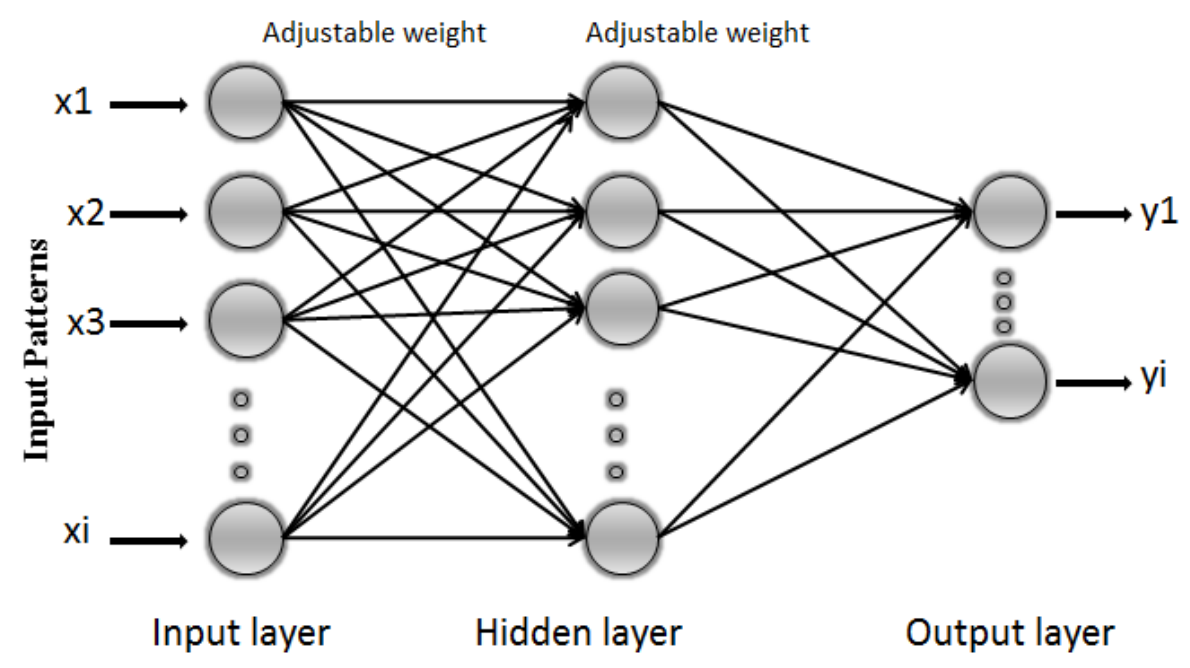

Figure 5.Diagram of MBP network.

\section{NARX Method}

NARX, which is depicted in Figure 6, has been used to predict the values of a time series, $y(t)$, from past values of that time series and past values of a second time series, $\mathrm{x}(\mathrm{t})$. In these experiments, the NARX used consists of numbers of hidden layers, numbers of delay lines (D) and one output neuron with two-layer feed forward networks. The standard Levenberg-Marquardt (LM) back-propagation algorithm is used to train the network with a learning rate close to 0.001. A method of regularization is used consisting of 1000 epochs and the regularization parameter used is $1.00 \mathrm{e}-05$. MATLAB code is used to run the ANN toolbox (nntool) generated by using the mode LM back-propagation (trainlm) (Howard \& Mark, 2000). 


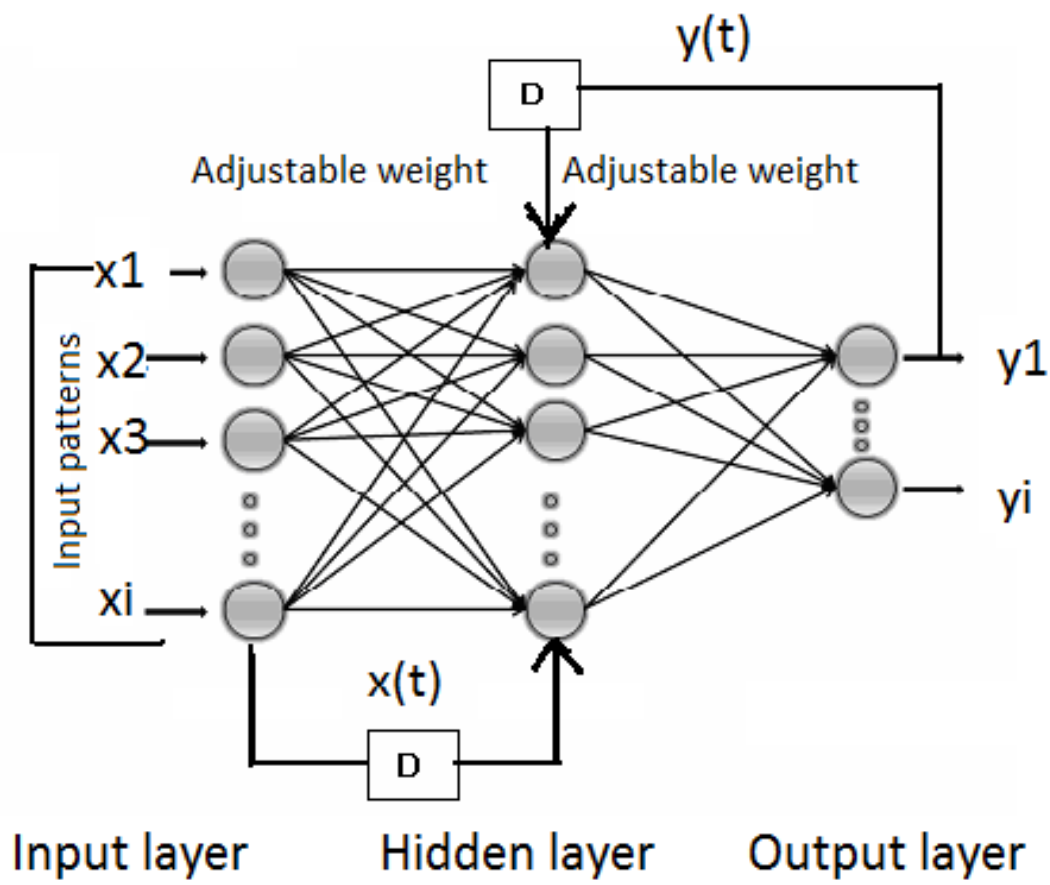

Figure 6.Diagram of NARX network

\section{RESULTS AND DISCUSSION}

The number of SMA wires applied has been considered as an output vector. Applied current, deflection and load are considered as the input vectors. All calculations of the neural network were made using MATLAB (Levenberg-Marquardt) and MBP open source code. The schematic diagrams of both models are displayed in Figures 7 and 8. Both the LM and MBP algorithms for training were applied to the network. The application randomly divides input vectors and target vectors into three sets, as follows: $80 \%$ are used for training: $10 \%$ are used to validate that the network is generalizing and to stop training before over-fitting. The last $10 \%$ are used as a completely independent test of network generalization.

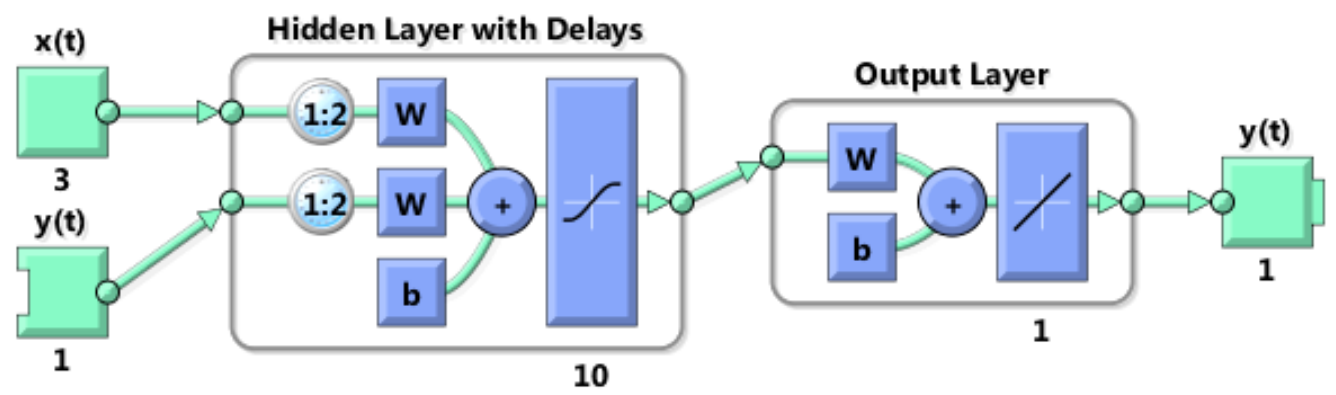

Figure 7.Example of NARX network with 10 hidden layers and 2 delays Timed by MATLAB 


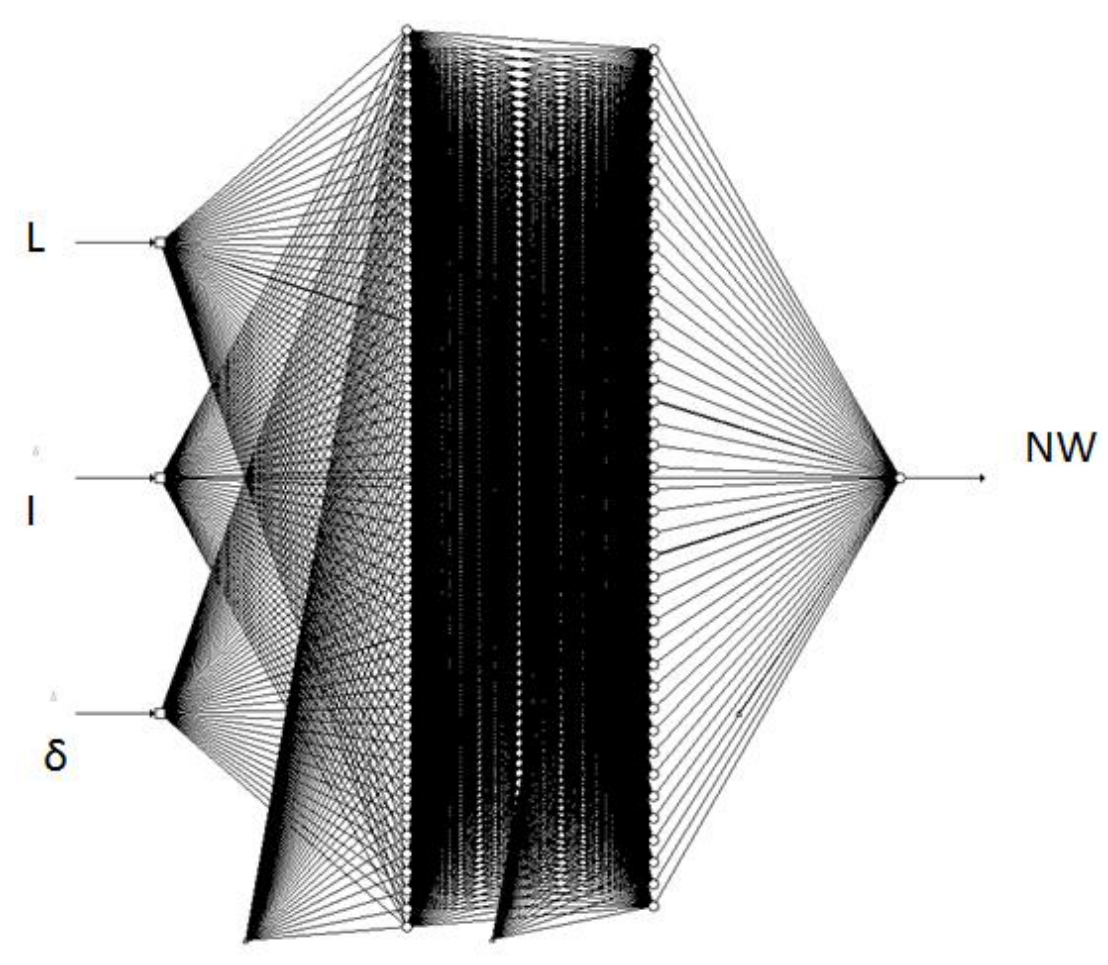

Figure 8.MBP diagram network with 50-40 hidden layers.

Data from experiments were collected to train the performance deflection number of wires in response to the load applied, deflection and the current applied. About 162 values of data were used for these networks. Table 1 shows that the NARX1 model yields the lowest MSE among the other ANN models and the fastest mode convergence training network. As can be seen from Table 2, the smallest values of MSE and the high values of $\mathrm{R}$ give us reason to consider the obtained NARX models to be adequate, being almost equal to unity.

Table 1.Predicting the deflection with respect to the number of wires using various models.

\begin{tabular}{|c|c|c|c|c|c|}
\hline Model & $\begin{array}{l}\text { Input } \\
\text { vector }\end{array}$ & $\begin{array}{l}\text { Output } \\
\text { vector }\end{array}$ & $\begin{array}{c}\text { Structure/No } \\
\text { hidden layer } \\
\text { neuron }\end{array}$ & $\begin{array}{c}\text { Epoch } \\
\text { (No. of } \\
\text { iterations) }\end{array}$ & $\begin{array}{c}\text { Mean } \\
\text { Square } \\
\text { Error } \\
(\mathrm{MSE}) \\
\end{array}$ \\
\hline MBP1 & $\overline{\mathrm{L}, \mathrm{I}, \mathrm{d}}$ & $\mathrm{NW}$ & $50-40$ & $1,273,277$ & 0.009999 \\
\hline MBP2 & $\mathrm{L}, \mathrm{I}, \mathrm{d}$ & NW & $50-40-30-20$ & 437,788 & 0.009997 \\
\hline NARX1 & $\mathrm{L}, \mathrm{I}, \mathrm{d}$ & NW & $\begin{array}{c}10 \text { delay time } \\
2\end{array}$ & 26 & 0.000308 \\
\hline NARX2 & $\mathrm{L}, \mathrm{I}, \mathrm{d}$ & NW & $\begin{array}{c}10 \text { delay time } \\
3\end{array}$ & 10 & 0.001542 \\
\hline NARX3 & $\mathrm{L}, \mathrm{I}, \mathrm{d}$ & NW & $\begin{array}{c}10 \text { delay time } \\
4\end{array}$ & 7 & 0.002337 \\
\hline
\end{tabular}


Table 2. Detailed results of the NARX model training.

\begin{tabular}{llll}
\hline & Target value & MSE & R \\
\hline Training & 130 & $8.12988 \mathrm{E}-5$ & $9.99145 \mathrm{e}-1$ \\
Validation & 16 & $3.08830 \mathrm{E}-4$ & $9.99289 \mathrm{e}-1$ \\
Testing & 16 & $3.49000 \mathrm{E}-3$ & $9.99597 \mathrm{e}-1$ \\
\hline
\end{tabular}

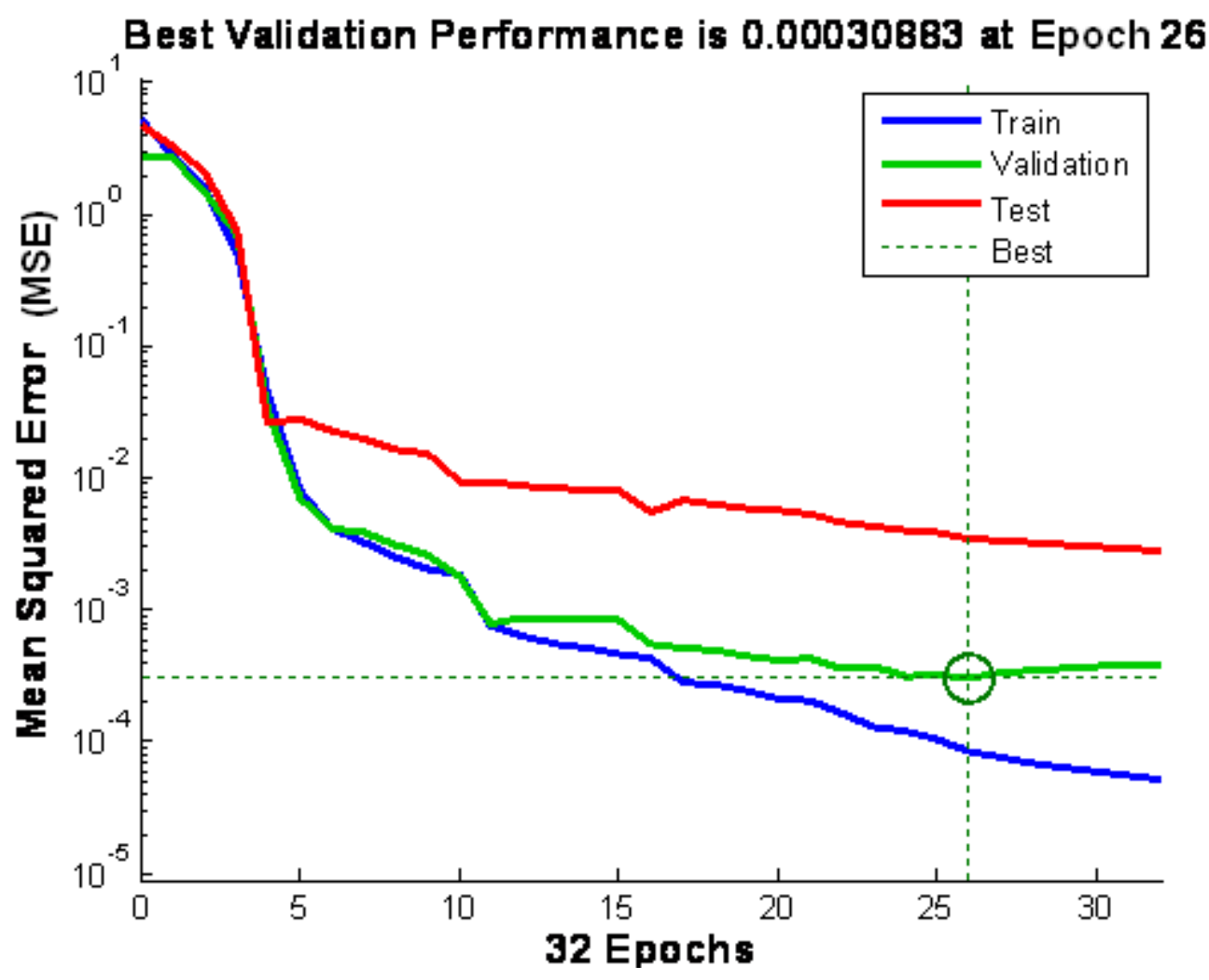

Figure 9.The network's performance.

The best validation performance is provided in Figure 9, which shows that the network's performance has improved during training. This performance is measured in terms of MSE and it is shown in log scale. It is evident that the MSE has decreased rapidly over the epochs while the network is trained. In this case, the results are reasonable, because of the following: the final mean-square error is very small, which means that the test set error and the validations set error have similar characteristics. In Figure 10, the training, test and validation data indicate a good fit of the curves. The validation and test results also show that the overall values are greater than 0.9 . 

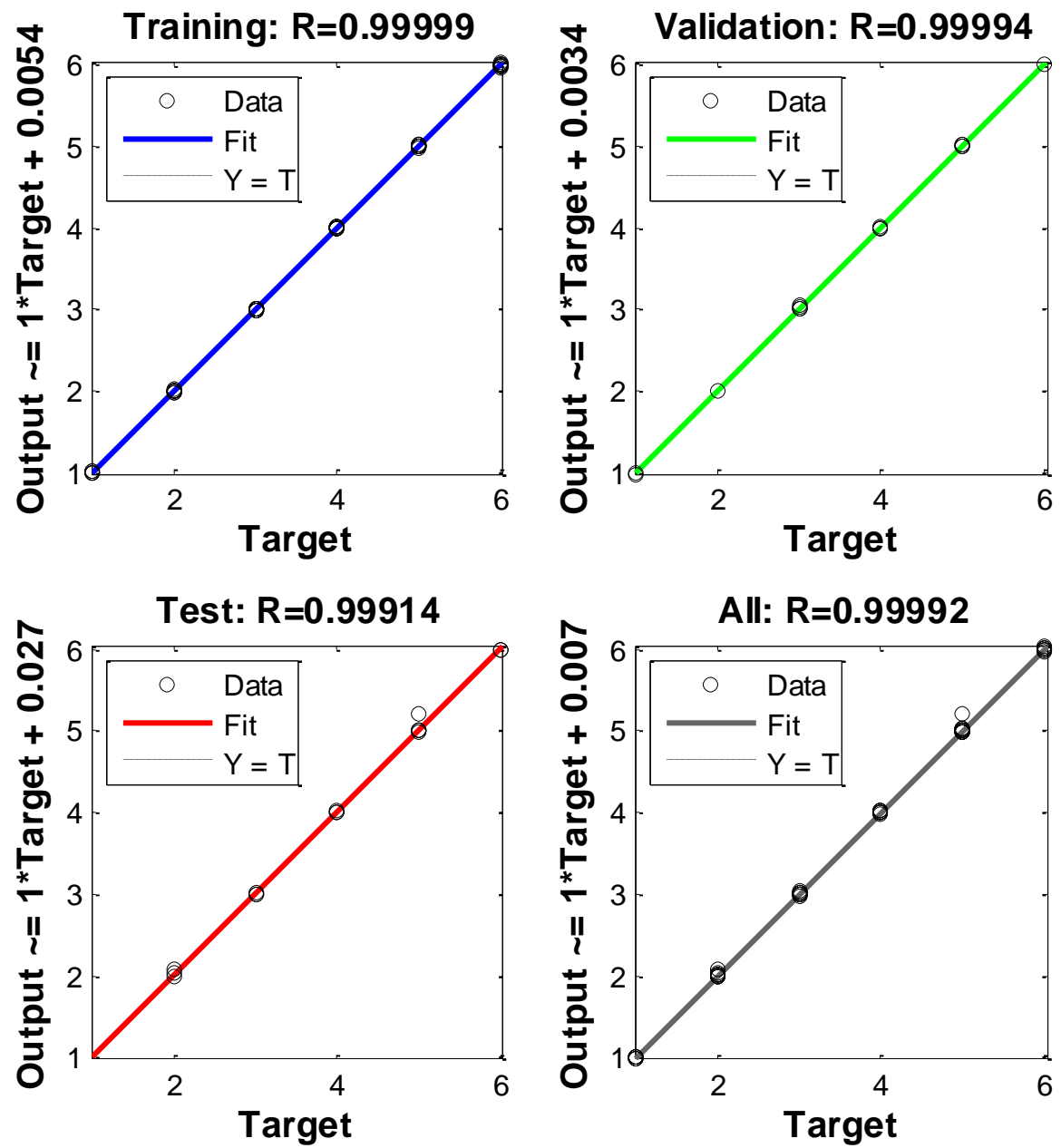

Figure 10.Regression analysis plot for the NARX.

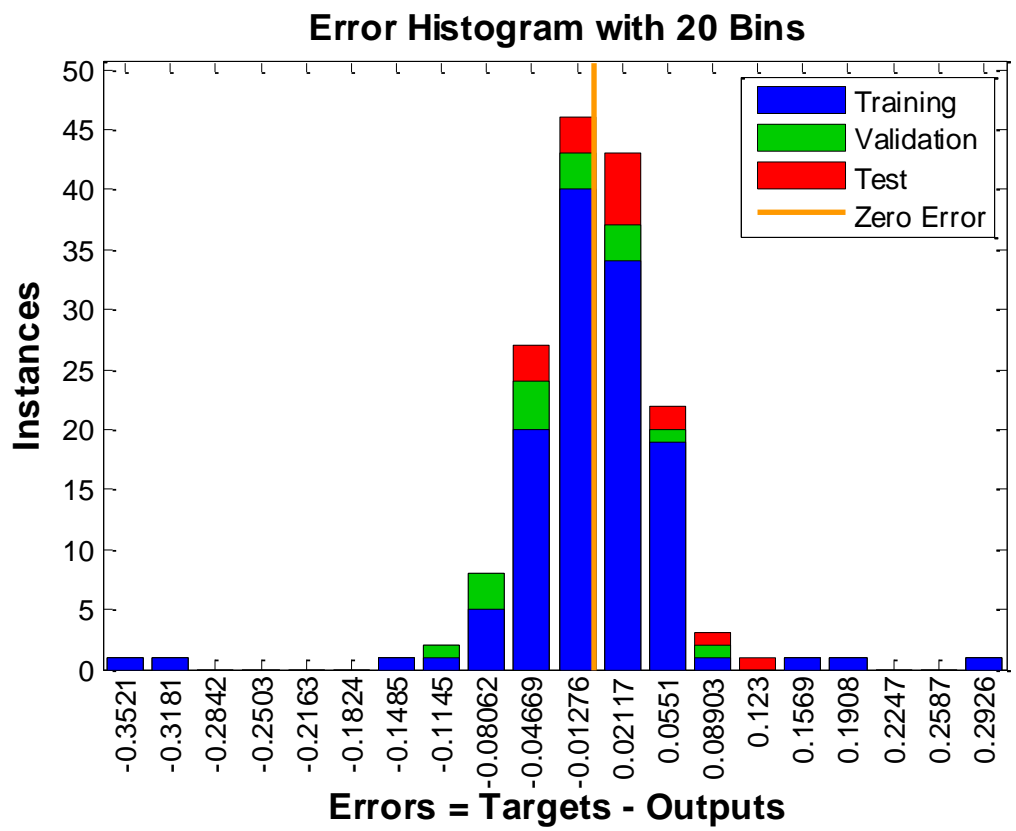

Figure 11.Error histogram of the NARX prediction model. 
Figure 11 shows how the error sizes are reasonably well distributed. Typically, when most errors are near zero, this indicates a better trained model. In this case however, it is confirmed that the network also has errors near zero. The correlation between input and error is provided in Figure 12. This figure illustrates how the errors are correlated with the input sequence. In a perfect prediction model, all the correlations should be zero. In this case, all of the correlations are within the confidence bounds around zero.
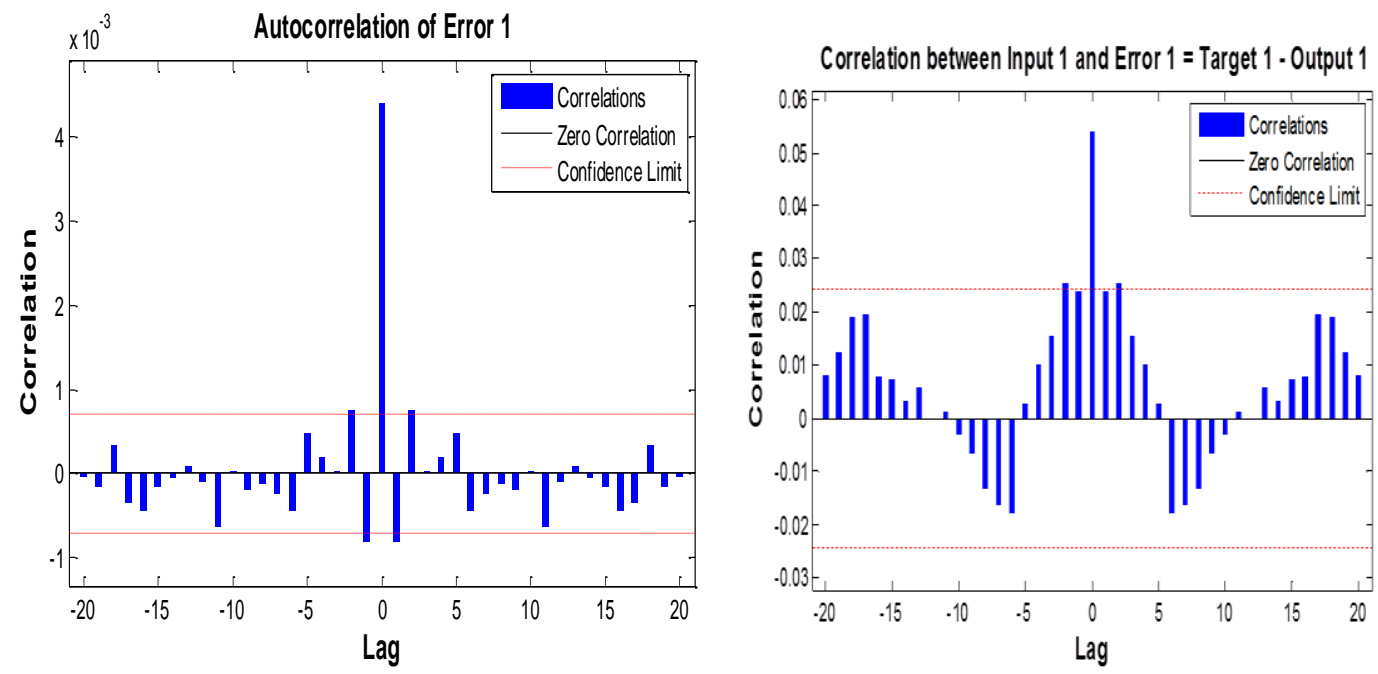

Figure 12.Auto-correlation of errors of NARX prediction model and correlation between input and output with respect to target function.

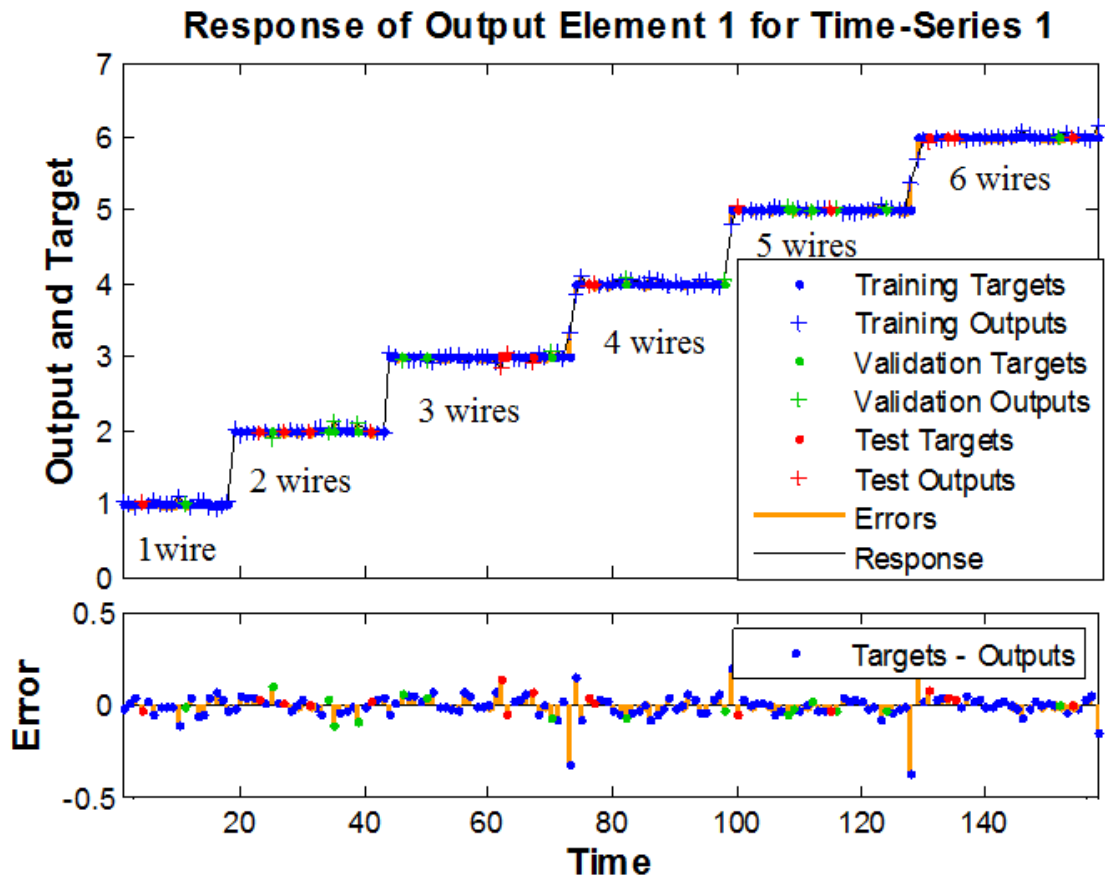

Figure 13.Response of NARX prediction model for performance deflection (trained by the Levenberg- Marquardt algorithm). 
The function of auto-correlation of errors is used to validate the network performance. Auto-correlation describes how the prediction errors are related in time. For the perfect model, there should be only one non-zero value of the auto-correlation at zero lag. This means that there is no correlation of prediction errors with each other. In this case, the correlations, except the one at zero lag, are within the $95 \%$ confidence limits. Based on the various diagnostics described up to now, it can be concluded that the model is adequate. Figure 13 confirms that the responses obtained from the NARX prediction model for the performance deflection are adequate, since the errors are quite small. For comparison, a similar shape also has been obtained, as shown in Figure 14.Comparing the predictions obtained based on both methods of network training, the NARX has improved the training network more than the MBP networks. In MBP, there are still network output errors with respect to the desired output network. Although the errors are not correlated with the input sequence, some of the correlations are not within the $95 \%$ confidence limit.

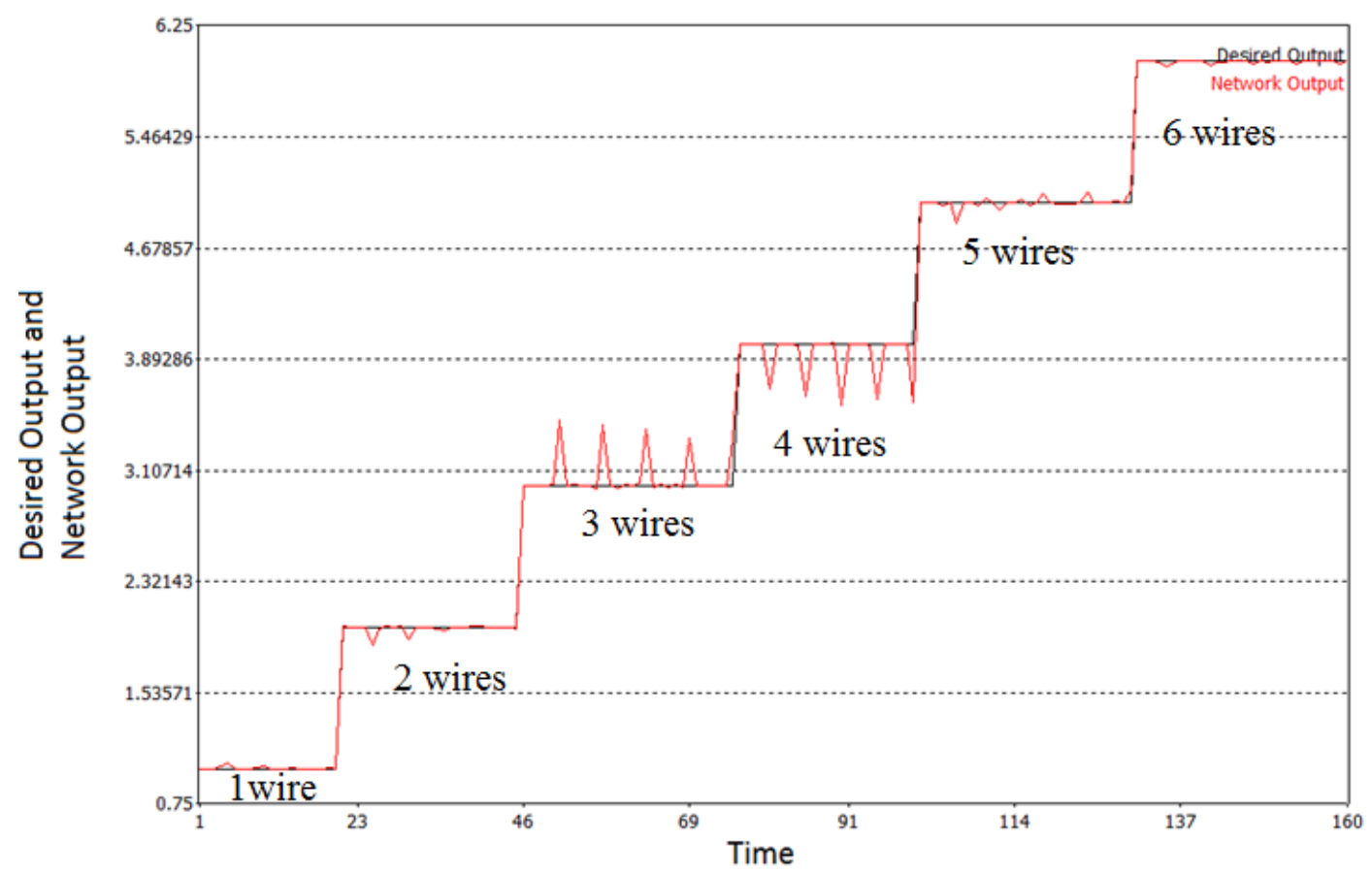

Figure 14.The desired output and network output by MBP using open source C code.

\section{CONCLUSIONS}

In this study, an ANN model with different network training methods was applied for predicting the amount of wire needed to restore the original shape and recover from deflection. The NARX and MBP algorithms were used for training of the network. The first conclusion of the paper is that although neural models may frequently suffer from a certain degree of inaccuracy, the results showed that the NARX model applied to the deflection of SMA proved the productivity and relation quality, at a lower computational expense. The NARX model was chosen since it resulted in the best performance according to MSE. Therefore, the NARX models have the potential to capture the dynamics of non-linear systems. The second conclusion is that the NARX models are mainly dependent on the applied architecture and training method. Within the context of the architecture, the behavior of NARX models mostly depends on the 
numbers of neurons in the hidden layers. Too many hidden neurons in the network cause over-fitting that, in turn, leads to poor predictions. Future modeling of the NARX is as follows: to model ANN 2 and ANN 3 which use deflection and applied current as the output vectors respectively.

\section{ACKNOWLEDGEMENT}

The authors would like to thank the UPM and MOHE of Malaysia for providing the research facilities and support in CEEFC of University of Southern Queensland, Australia.

\section{REFERENCES}

Abaqus. (2012). Abaqus/cae release note 6.12.

Aeyzarq Muhammad Hadzreel, M. R., \& Siti Rabiatull Aisha, I. (2013). Effect of reinforcement alignment on the properties of polymer matrix composite. Journal of Mechanical Engineering and Sciences, 4, 548-554.

Gayan, C. K., Jayantha, A. E., Hao, W., \& Lau, K. T. (2013). Prediction of obsolete fbg sensor using ann for efficient and robust operation of shm systems. Key Engineering Materials, 558, 546-553.

Howard, D., \& Mark, B. (2000). Neural network toolbox for computation, visualization and programming-user's guide The MathWorks, I. (Ed.) pp. 323.

Khan, M. A. R., Rahman, M. M., Kadirgama, K., \& Bakar, R. A. (2012). Artificial neural network model for material removal rate of ti-15-3 in electrical discharge machining. Energy Education Science and Technology Part A: Energy Science and Research, 29(2), 1025-1038.

Khan, M. A. R., Rahman, M. M., Kadirgama, K., Maleque, M. A., \& Bakar, R. A. (2011). Artificial intelligence model to predict surface roughness of ti-15-3 alloy in edm process. World Academy of Science, Engineering and Technology, 74, 198-202.

M. Khairul Zaimy, A. G., Zafiah, A., Rus, M., Ab Latif, N., \& Nurulsaidatulsyida, S. (2013). Mechanical and thermal properties of waste bio-polymer compound by hot compression molding technique. Journal of Mechanical Engineering and Sciences, 5, 582-591.

Noel, L., \& Bernardete, R. (2001). Hybrid learning multi neural architecture. IEEE International Joint Conference on Neural Networks, 4, 2788-2793.

Noel, L., \& Bernardete, R. (2003). An efficient gradient-based learning algorithm applied to neural networks with selective actuation neurons. Neural Parallel and Scientific Computations, 11, 253-272.

Nolet, S. C. (2011). Composite wind blade engineering and manufacturing. Indepedent activities period mini-course. from http://web.mit.edu/windenergy/windweek/ Presentations/Nolet_Blades.pdf.

Peter, J. S., \& Richard, J. C. (2012). Wind turbine blade design: Review. Energies, 5, 3425-3449

Rahman, M. M., Mohyaldeen, H. M., Noor, M. M., Kadirgama, K., \& Bakar, R. A. (2011). Linear static response of suspension arm based on artificial neural network technique Advanced Materials Research, 213, 419-426.

Sapuan, S. M., \& Iqbal, M. M. (2010). Composite materials technology : Neural network applications: CRC Press Taylor and Francis Group. 
Sorensen, B. F., Jørgensen, E., Christian, P. D., \& Jensen, F. M. (2004). Improved design of large wind turbine blade of fibre composites based onstudies of scale effects (phase 1) Ris $\phi-R-1390$ (En) Denmark.

Srihari, P. V., Govindarajulu, K., \& Ramachandra, K. (2010). A method to improve reliability of gearbox fault detection with artificial neural networks. International Journal of Automotive and Mechanical Engineering, 2, 221-230.

Supeni, E. E., Epaarachchi, J. A., Islam, M. M., \& Lau, K. T. (2012a). Design and analysis of a smart composite beam for small wind turbine blade construction. Paper presented at the The Southern Region Engineering Conference (SREC) USQ, Toowoomba, Australia.

Supeni, E. E., Epaarachchi, J. A., Islam, M. M., \& Lau, K. T. (2012b). Development of smart wind turbine blades. Paper presented at the The 8th Asian-Australasian Conference on Composite Materials (AACM-8), (KLCC),Kuala Lumpur, Malaysia. 